Chapter III

STELLAR AND PLANETARY DYNAMOS 


\title{
Mechanisms of planetary and stellar dynamos
}

\author{
CNRS, Equipe MAG (ENS-IPGP), \\ LRA, Département de Physique, \\ Ecole Normale Supérieure, \\ 24 rue Lhomond, 75005 Paris, France \\ email: dormy@phys.ens.fr
}

Emmanuel Dormy, Ludovic Petitdemange \& Martin Schrinner

\begin{abstract}
We review some of the recent progress on modeling planetary and stellar dynamos. Particular attention is given to the dynamo mechanisms and the resulting properties of the field. We present direct numerical simulations using a simple Boussinesq model. These simulations are interpreted using the classical mean-field formalism. We investigate the transition from steady dipolar to multipolar dynamo waves solutions varying different control parameters, and discuss the relevance to stellar magnetic fields. We show that owing to the role of the strong zonal flow, this transition is hysteretic. In the presence of stress-free boundary conditions, the bistability extends over a wide range of parameters.
\end{abstract}

Keywords. MHD, Dynamo, Magnetic fields.

\section{Introduction}

Magnetic fields of low-mass stars and planets are thought to originate from self-excited dynamo action in their convective interiors. The accepted theory, known as dynamo theory, describes the transfer from kinetic to magnetic energy as an instability process. Above a given threshold electrical currents, and thus magnetic fields, are amplified by a turbulent flow of a conducting fluid.

Observations of the magnetic fields produced by direct numerical simulations (DNS) of dynamo action appear to fall into two categories: fields dominated by large-scale dipoles (such as the Earth and a fully convective star), and fields with smaller-scale and non-axisymmetric structures (such as the Sun). Two kinds of different temporal behaviour have also been identified: very irregular polarity reversals (as in the Earth), and quasi-periodic reversals (as in the Sun). Since the Earth and the Sun provide the largest database of magnetic field observations, these objects have been well studied and described in terms of alternative physical mechanisms: the geodynamo involves a steady branch of the dynamo equations, with fluctuations and possibly polarity reversals, whereas the solar dynamo takes the form of a propagating dynamo wave. The signature of this wave at the Sun's surface yields the well-known butterfly-diagram (Sunspots preferentially emerge at a latitude that is decreasing with time during the solar cycle).

\section{Bifurcation diagrams}

Let us start by considering the origin of the Earth's magnetic field, which remains a challenging issue for physicists. We consider the magnetohydrodynamic system of equations in a rotating spherical shell. The problem can be described in its simpler form by a set of coupled partial differential equations written in the classical Boussinesq limit The 

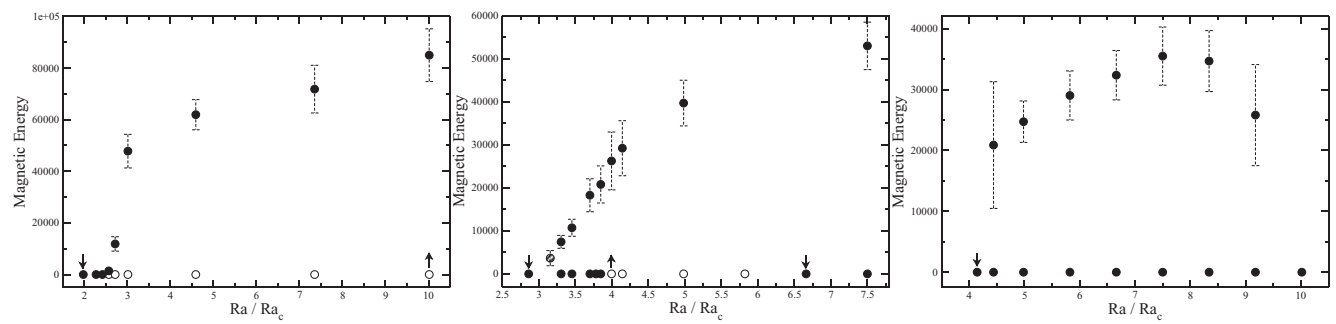

Figure 1. Mean magnetic energy as a function of the Rayleigh number. Error bars indicate the standard deviation of energy fluctuations around the mean value. All simulations are performed for $\mathrm{E}=3 \cdot 10^{-4}$. The bifurcation is found to be super-critical for $\mathrm{Pm}=6$ (left), sub-critical for $\mathrm{Pm}=3$ (middle), and yields an isola diagram for $\mathrm{Pm}=1.5$ (right) [After Morin \& Dormy (2009)].

governing equations can then be written in non-dimensional form

$$
\begin{aligned}
& \mathrm{E}_{\eta}\left[\partial_{t} \mathbf{u}+(\mathbf{u} \cdot \boldsymbol{\nabla}) \mathbf{u}\right]=-\boldsymbol{\nabla} \pi+\mathrm{E} \Delta u-2 \mathbf{k} \times \mathbf{u} \\
&-\operatorname{Ra} T \mathbf{g}+(\boldsymbol{\nabla} \times \mathbf{B}) \times \mathbf{B}, \\
& \partial_{t} \mathbf{B}=\boldsymbol{\nabla} \times(\mathbf{u} \times \mathbf{B})+\Delta \mathbf{B} \\
& \partial_{t} T+(\mathbf{u} \cdot \boldsymbol{\nabla}) T=\mathrm{q} \Delta T \\
& \boldsymbol{\nabla} \cdot \mathbf{u}=\boldsymbol{\nabla} \cdot \mathbf{B}=0
\end{aligned}
$$

where

$$
\mathrm{E}=\frac{\nu}{\Omega L^{2}} ; \mathrm{Pm}=\frac{\nu}{\eta} ; \mathrm{E}_{\eta}=\frac{\mathrm{E}}{\mathrm{Pm}} ; \mathrm{q}=\frac{\kappa}{\eta} ; \mathrm{Ra}=\frac{\alpha g \Delta T L}{\nu \Omega} .
$$

The state of this system is fully characterised by four independent controlling parameters. The Ekman number E, which can be interpreted as measuring the ratio of the period of rotation (the length of the day in the case of the Earth) to a typical viscous timescale, this number is extremely small in the case of the Earth's core (the Earth, as the Sun, is in rapid rotation). The magnetic Prandtl number Pm, measuring the ratio of a typical ohmic timescale to viscous timescale, it is a characteristic of the fluid and is minute for all liquid metals (including liquid iron in the Earth's core). The Roberts number q, also characterizing the fluid and which compares a thermal timescale to the ohmic timescale, this number is comparable with Pm. Finally the Rayleigh number Ra, which measures a ratio of driving forces to forces slowing down the motion. Its value is difficult to assess in a simple Boussinesq model. This will be the most obvious controlling parameter, which needs to be varied to investigate dynamo properties.

We investigated in Morin \& Dormy (2009) the nature of the dynamo bifurcation in a configuration applicable to the Earth's liquid outer core, i.e. in a rotating spherical shell with thermally driven motions. We show that the nature of the bifurcation, which can be either super-critical or sub-critical or even take the form of isola (or detached lobes) strongly depends on the parameters. This dependence is described in a range of parameters numerically accessible (which unfortunately remains remote from geophysical application), and we show how the magnetic Prandtl number and the Ekman number control these transitions.

We have studied in Morin \& Dormy (2009) different bifurcations obtained for Ekman number values ranging from $10^{-3}$ to $10^{-4}$, magnetic Prandtl number values from 0.67 to 6 and Rayleigh number values from $\mathrm{Ra} \simeq 2 \times \mathrm{Ra}_{c}$ to $\mathrm{Ra} \simeq 10 \times \mathrm{Ra}_{c}$. In this parameter regime, for a given Ekman number, a super-critical bifurcation is obtained for 

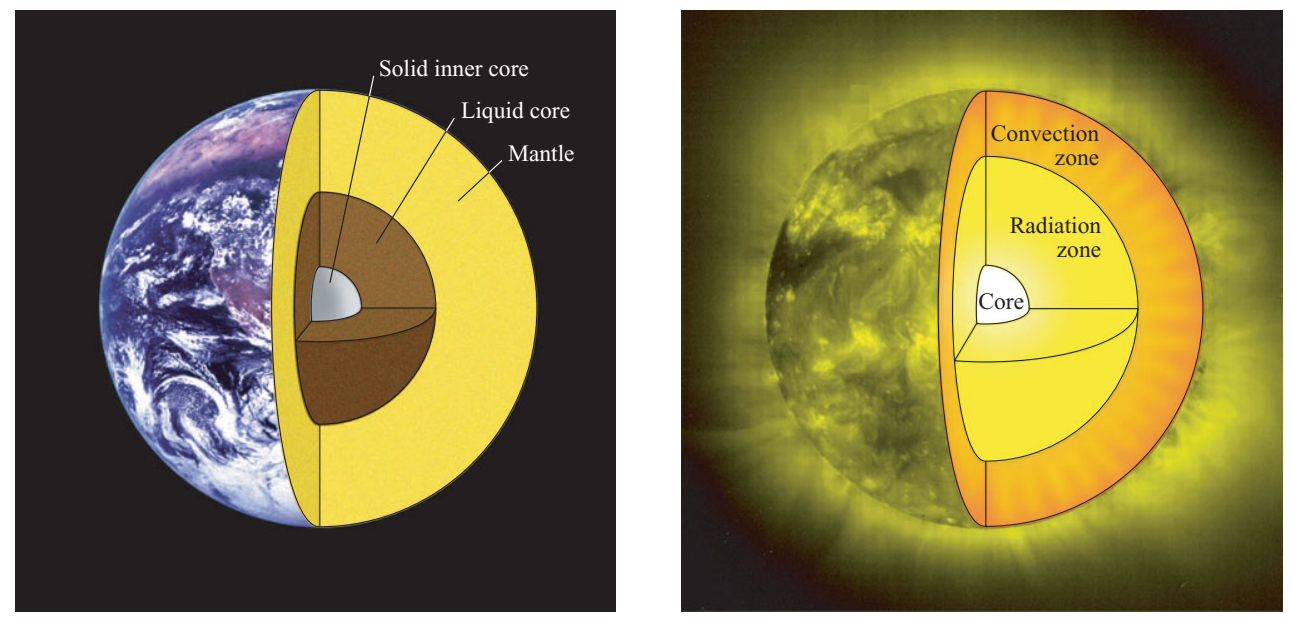

Figure 2. Comparison of the Earth and Sun interior. The first striking difference regarding dynamo action is the aspect ratio of the dynamo region. The radiative zone of the Sun occupies a much larger fraction of the conducting region than the solid inner core of the conducting core [Figures from Dormy \& Soward (2007)].

a sufficiently high value of the magnetic Prandtl number. By decreasing its value, it is possible to obtain a sub-critical bifurcation, which may exhibit unusual features, such as re-stabilization of the purely hydrodynamical state. An unstable branch therefore must exist, it could be connected, for larger values of the Rayleigh number, to the stable branch corresponding to dynamo solutions. If the magnetic Prandtl number is further decreased, the range of Rayleigh number for which the non dynamo solution is unstable vanishes. An isola is then obtained, in this situation the purely hydrodynamical solution is always stable. The very same sequence is obtained by increasing $\mathrm{E}$ at fixed Pm, as the dipolar domain shifts towards lower values of $\mathrm{Pm}$ as $\mathrm{E}$ is decreased. We refer the reader to Morin \& Dormy (2009) for further discussion on these aspects.

\section{From the Earth to the stars...}

Because of their very different natures (liquid metal in one case, plasma in the other), planetary and stellar magnetic fields are studied by different communities. Nondimensional numbers controlling the dynamics of the Earth and the Sun, for example, do significantly differ (see Zhang \& Schubert, 2006; Tobias \& Weiss, 2007). As a practical matter however, the techniques as well as the typical parameters used in numerical studies of these two systems are surprisingly similar. To some extent this is due to the restricted parameter space available to present day computations. The parameter regime numerically accessible is rather remote from the actual objects. For planetary dynamos the main discrepancy relies in the rapid rotation in the momentum equation (characterized by the Ekman number), whilst for stellar dynamos it relies in solving the induction equation with weak resistive effects (characterized by high values of the magnetic Reynolds number).

The relative success of numerical models to reproduce some of the key characters to both geo (e.g. Glatzmaier \& Roberts, 1995) and solar (e.g. Gilman, 1983 and Browning et al., 2006) magnetic fields have lead us to argue that the aspect ratio of the dynamo region (i.e. the radius ratio of the inner bounding sphere to the outer bounding sphere) could be an essential parameter. Indeed, in the Earth, the inert solid inner core extends 
to some $35 \%$ of the core radius, whereas in the Sun, the radiative zone fills $70 \%$ of the solar radius (see Fig. 2). One expects the convective zones of stars and planets to have all possible intermediate aspect ratios, even extending to fully convective spheres.

In Goudard \& Dormy 2008, we showed that by varying the aspect ratio, we could observe a sharp transition from a dipole dominated large scale-magnetic field to a cyclic dynamo with a weaker dipole. Indeed in our simulations at fixed parameters, the strongly dipolar solution becomes unstable with an increase of the aspect ratio above 0.65 . For this value -close to that of the Sun- we observed that the strong dipole is first maintained and then rapidly weakens, while dynamo action continues in a different form: that of a wavy solution with quasi-periodic reversals (Fig. 3), reminiscent of some aspects of the solar magnetic field behavior. This indicated that the geometry of the dynamo region could severely constrain the existence of the dipole dominated solution.

Because of the strong symmetry of the convective flows influenced by the rapid rotation of the planet or the star, it is well known that two independent families of solutions can exist: with dipole symmetry (antisymmetric with respect to the equator) and quadrupole symmetry (symmetric with respect to the equator). We have indeed observed these two families in our fully three dimensional simulations (Fig. 4).

Figure 5 shows the azimuthally averaged field for some of our fully $3 \mathrm{D}$ simulations. The Earth-like mode is represented for aspect ratios of 0.45 and 0.6 ( $a$ and b). The active dynamo region lies outside the tangent cylinder, it therefore gets increasingly constrained as the inner sphere in increased. The dipole eventually drops for large aspect ratio, when the volume outside the tangent cylinder becomes too small.

Let us note that the above solutions (Goudard \& Dormy, 2008) have since then been reproduced using an independently written code by Simitev et al. (2010) and Simitev \& Busse (2012).

We shown that the steady dynamo branch can be replaced, at larger aspect ratio, by an oscillatory dynamo mode. Comparison with reduced parametrized models can help interpret this transition to the solar-like mode. A strong zonal wind develops, in the Solar-like regime. This prompted us to suggest a transition from an $\alpha^{2}$ type to a dynamo of the $\alpha \Omega$ type as the aspect ratio is increased. We shall see that this requires a careful analysis.

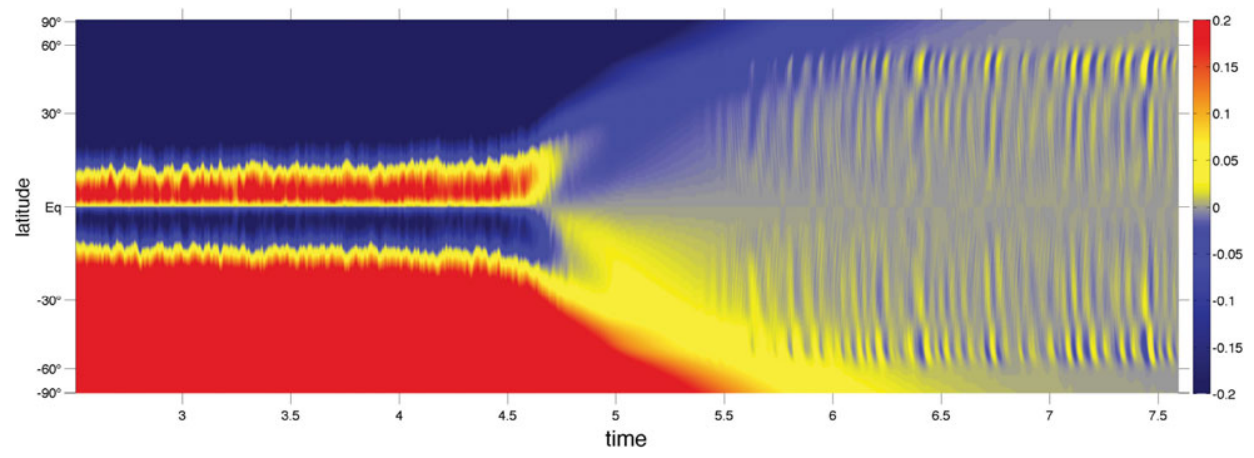

Figure 3. Time evolution of the radial magnetic field averaged in longitude (for an aspect ratio of 0.65). The initial dipole field survives for a few diffusion times, and then vanishes to yield a butterfly diagram [After Goudard \& Dormy (2008)]. 


\section{Dynamo Mechanisms}

Using the test-field method introduced in Schrinner et al. (2007), Schrinner (2011), we could compute mean-field dynamo coefficients in Schrinner et al. (2011). These coefficients have been used in a mean-field calculation in order to explore the underlying dynamo mechanism.

The evolution of the magnetic field is cyclic. In Fig. 6 (left), the azimuthally averaged radial magnetic field is represented at the outer shell boundary as a function of time (the so-called butterfly diagram). A dynamo wave migrates away from the equator until it reaches mid-latitudes where the inner core tangent cylinder intersects the outer shell boundary. The magnetic field looks rather small scaled and multipolar. This is confirmed by the magnetic energy spectrum which is essentially white, except for a negligible dipole contribution. Furthermore, the magnetic field is weak, as expressed by an Elsasser number of $\Lambda=B_{\mathrm{rms}}^{2} /(\mu \rho \eta \Omega)=0.13$.

The kinematically advanced tracer field grows slowly in time, i.e. the model under consideration is kinematically unstable according to the classification by Schrinner et al. (2010). But, deviations of the tracer field from the actual field are hardly noticeable in the field morphology. Moreover, the very same dynamo wave persists in the kinematic calculation (see also Goudard \& Dormy, 2008), as visible in figure 6 (middle). Note that the tracer field in figure 6 has evolved from random initial conditions. This time
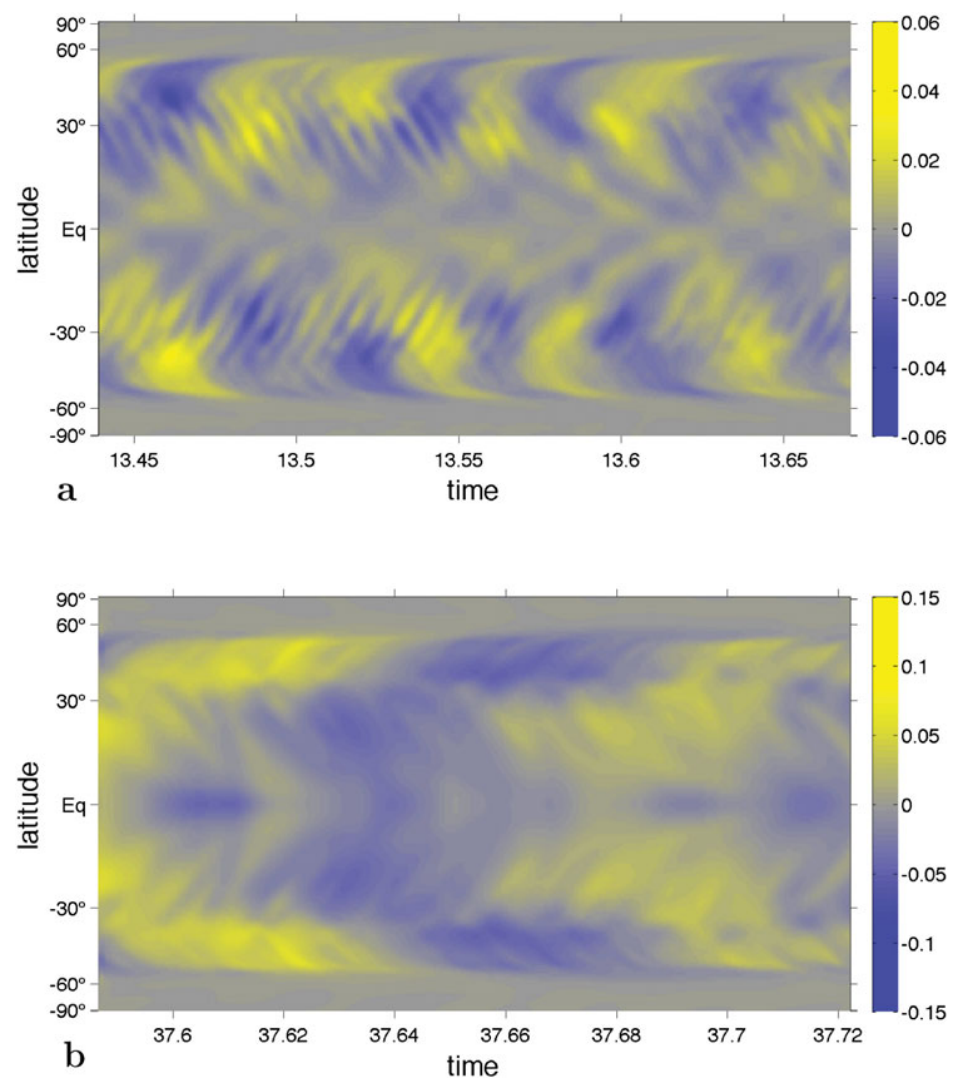

Figure 4. Time evolution of the zonal average of the azimuthal magnetic field below the surface of the model, for an aspect ratio of 0.65: the antisymmetric (a) and symmetric (b) solutions [After Goudard \& Dormy (2008)]. 
dependent mode offers a remarkable test of the mean-field coefficient derived from the test-field approach.

A mean-field calculation based on the dynamo coefficients derived using the test-field approach and the mean flow determined from the self-consistent model is presented in figure 6 . The fastest growing eigenmodes form a conjugate complex pair and give rise to a dynamo wave which compares nicely with the direct numerical simulations.

The influence of the differential rotation can be suppressed in the kinematic calculation of the tracer field without changing any other component of the flow. A butterfly diagram resulting from a kinematically advanced field is presented in figure 7 (left). Interestingly, the evolution of the magnetic field is again cyclic. The right chart of figure 7 presents the butterfly diagram corresponding to the fastest growing eigenmodes of the resulting $\alpha^{2}$-dynamo.

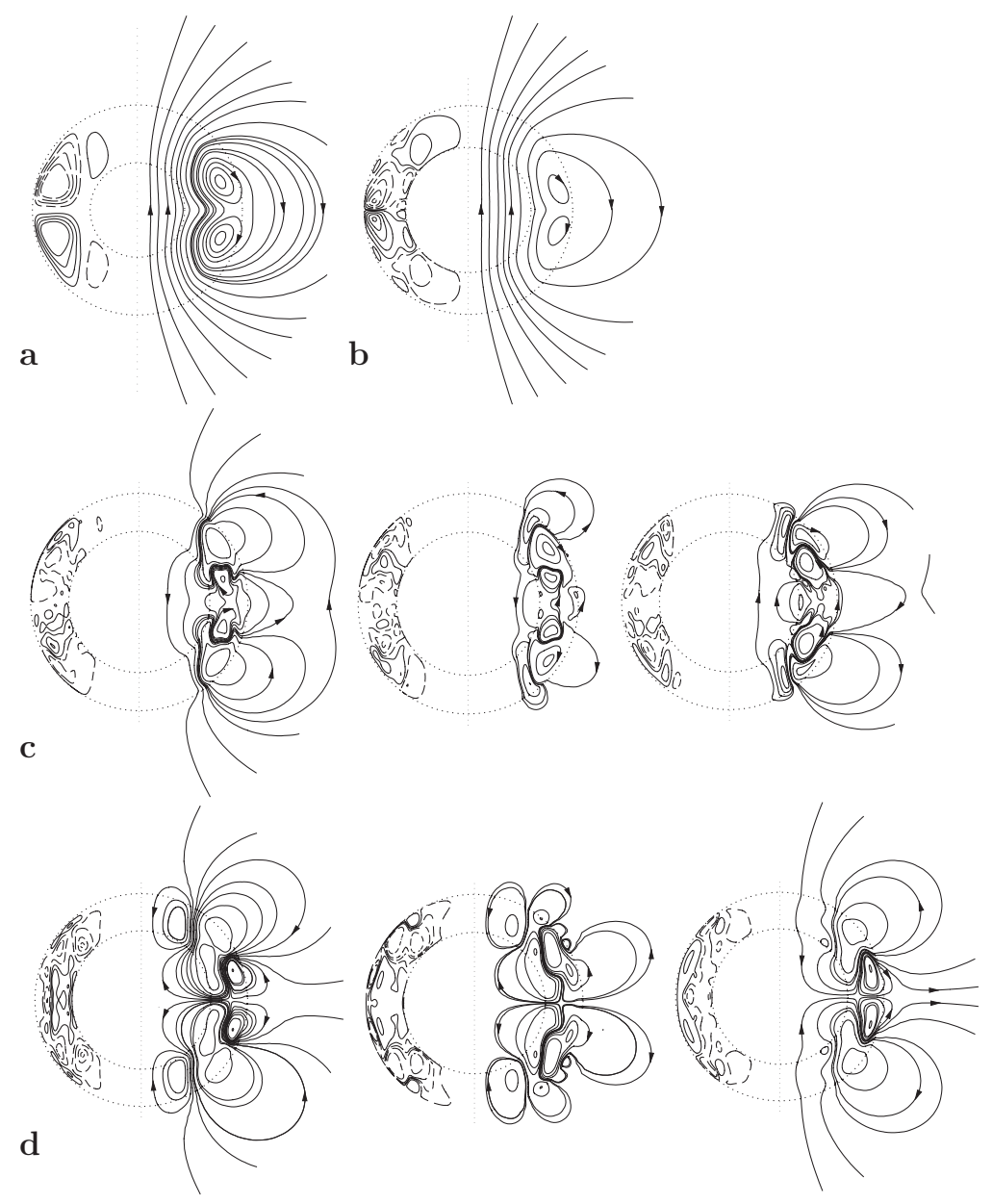

Figure 5. The zonal average of the magnetic field in our 3D simulations. Contours of the toroidal (east-west) part of the field are plotted in the left hemisphere and lines of force of the meridional (poloidal) part of the field plotted in the right hemisphere. The aspect ratio is increased from 0.45 (a) to 0.6 (b) and to 0.65 (c-d). The sequence of dynamo waves is represented for the antisymmetric mode (c) and symmetric mode (d). It is similar in nature to that produced by parametrized models, see Roberts (1972) [After Goudard \& Dormy (2008)]. 

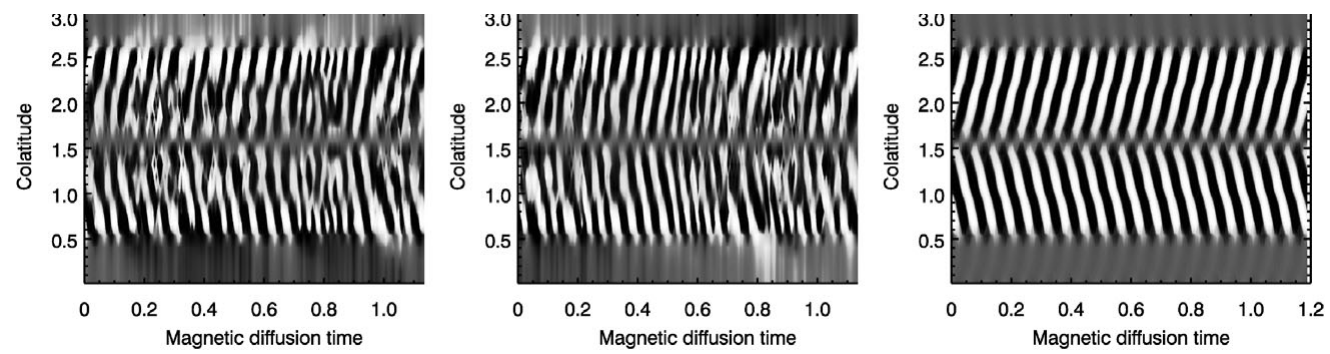

Figure 6. Azimuthally averaged radial magnetic field at the outer shell boundary varying with time (butterfly diagram) resulting from a self-consistent calculation (left), kinematic calculation (middle) and mean-field calculation (right). The contour plots have been normalised by their maximum absolute value at each time step considered [After Schrinner et al. (2011)].
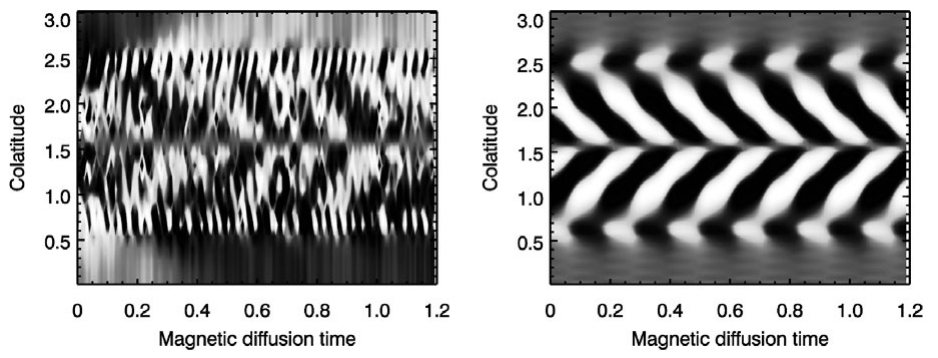

Figure 7. Azimuthally averaged radial magnetic field at the outer shell boundary varying with time (butterfly diagram) resulting from a kinematic calculation with subtracted $\Omega$-effect (left) and a corresponding mean-field calculation (right). The contour plots are presented as in figure 6 [After Schrinner et al. (2011)].

A particular dynamo mechanism does not seem to be responsible for the occurrence of periodically time-dependent magnetic fields. It turns out, that the influence of the largescale radial shear (the $\Omega$-effect), is not necessary for cyclic field variations. Instead, the action of small-scale convection happens to be essential. For the model presented here, small convective length scales are forced by a thin convection zone. Further investigations are needed to assess whether our finding is representative for a wider class of oscillatory models.

This study revealed that the oscillatory dynamo model under consideration is of the $\alpha^{2} \Omega$-type. Although the rather strong differential rotation present in this model influences the magnetic field, the $\Omega$-effect alone is not responsible for its cyclic time variation. If the $\Omega$-effect is suppressed the resulting $\alpha^{2}$-dynamo remains oscillatory. Surprisingly, the corresponding $\alpha \Omega$-dynamo leads to a non-oscillatory magnetic field. The simpler assumption of an $\alpha \Omega$-mechanism therefore does not explain satisfactorily the occurrence of magnetic cycles.

\section{Dipole breakdown and bistability}

We then investigated in Schrinner et al. (2012) over seventy three-dimensional, selfconsistent dynamo models obtained by direct numerical simulations. The control parameters, the aspect ratio and the mechanical boundary conditions have been systematically varied to build up this sample of models.

Both, strongly dipolar and multipolar models have been obtained. We could show in Schrinner et al. (2012) that these dynamo regimes can in general be distinguished by the ratio of a typical convective length-scale to the Rossby radius (see Schrinner et al., 2012 


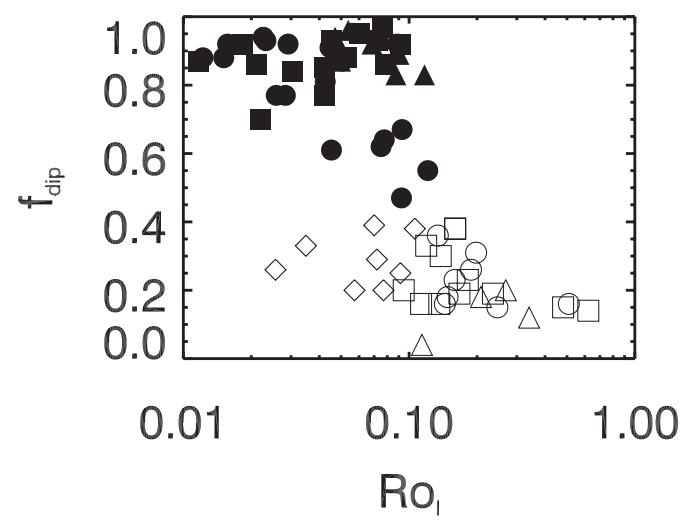

Figure 8. Relative dipole field strength versus the local Rossby number for all 72 models. Filled symbols stand for models dominated by a dipole field, open symbols denote multipolar models. The symbol shape indicates different types of mechanical boundary conditions: circles mean no-slip conditions at both boundaries, triangles are models with a rigid inner and and a stress-free outer boundary, and squares stand for models with stress-free conditions at both boundaries. Simulations started from a weak magnetic field (diamonds) [After Schrinner et al. (2012)].

for a precise definition of $\mathrm{Ro}_{\ell}$ ). Models with a predominantly dipolar magnetic field were obtained, if the convective length scale is at least an order of magnitude larger than the Rossby radius (see Fig. 8).

Moreover, we have highlighted the role of the strong shear associated with the geostrophic zonal flow for models with stress-free boundary conditions. In this case the above transition disappears and is replaced by a region of bistability for which dipolar and multipolar dynamos co-exist (see again Fig. 8).

There is a strong correlation between the topology and the time dependence of the magnetic field in dynamo models. Sudden polarity reversals or oscillations of the magnetic field do not occur in dipole dominated models in the low Rossby number regime. Conversely, reversals and oscillations are frequent in non-dipolar models with $\mathrm{Ro}_{\ell}>0.1$ as well as in models with lower local Rossby numbers with stress-free boundary conditions belonging to the multipolar branch.

Whether non-dipolar models exhibit fairly coherent oscillations or irregular reversals of the magnetic field strongly depends on the magnetic Reynolds number. Coherent oscillatory solutions of the induction equation are most clearly visible in so-called butterfly diagrams; contours of the azimuthally averaged radial magnetic field at the outer boundary are plotted versus time and colatitude.

Figure 10 gives an illustration of this transition and at larger $\mathrm{Rm}$ is much less periodic and a cycle period cannot be identified. Dynamo models (in the non-dipolar regime) at higher magnetic Reynolds number exhibit even less temporal coherence. Following this somewhat arbitrary and qualitative criterion, we find that non-dipolar dynamos of our sample with $\mathrm{Rm} \lesssim 200$ generate magnetic fields which vary periodically in time. The lower the magnetic Reynolds number, the more coherent is the time variability of the magnetic field. This has important implications on the applicability of these models to stars as the Sun with a well defined cycle.

It is interesting to ponder on the transitions between the dipolar and multipolar branch for stress-free models when one single control parameter is varied. The two branches are illustrated in Fig. 9 for a fixed Ekman number of $E=10^{-4}$ and magnetic Prandtl number of $P m=1$. For both branches, the local Rossby number increases with increasing 


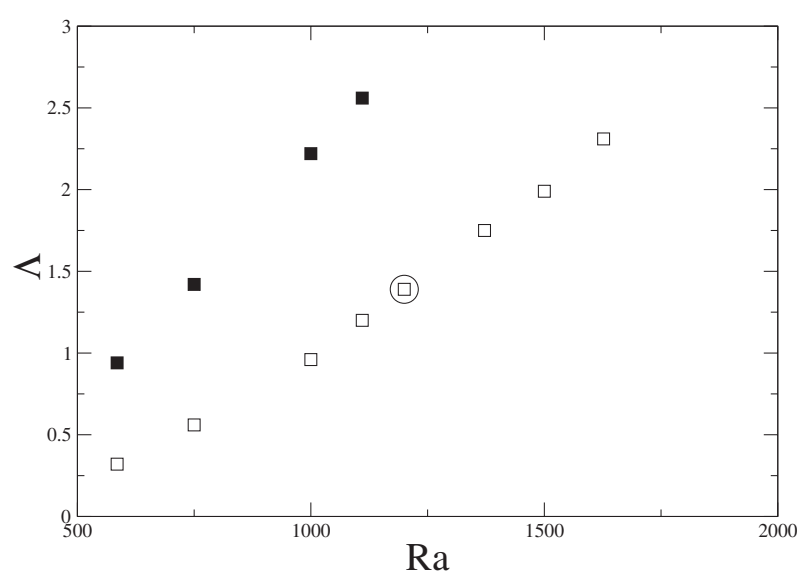

Figure 9. Evolution of the magnetic field strength, measured by the Elsasser number, for both branches as the Rayleigh number is varied at fixed Ekman and magnetic Prandtl numbers $(\mathrm{E}=$ $10^{-4}$ and $\left.\mathrm{Pm}=1\right)$. Filled symbols stand for models dominated by a dipole field, open symbols denote multipolar models. These models used stress-free conditions at both boundaries [After Schrinner et al. (2012)].

Rayleigh numbers. If the Rayleigh number is increased from $R a=1110$ on the dipolar branch to $R a=1200$, the relative dipole field strength collapses (the local Rossby number crosses the $\mathrm{Ro}_{\ell} \sim 0.1$ boundary). The multipolar field configuration then appears to be the only stable solution (circle on the figure) and a hysteretic behavior is observed if the Rayleigh number is decreased from this state.

\section{Mean-field, Boussinesq and Anelastic: a hierarchy of models}

We have presented here several numerical developments relying on a simple Boussinesq dynamo model. Assuming a constant reference density is clearly a strong simplification of the governing equations. This approximation is fairly reasonable for experimental setups, but harder to justify on the large scale of planetary or stellar interiors. When the reference state is stratified under its own weight, but the fluid velocity remains small compared to the sound speed, it is safer to rely on an anelastic formalism (different approximations can be designated by this name).

We are thus left with a hierarchy of tools in order to investigate the magnetic field generation in planets and stars.

The first approach, which is also the lightest in terms of computational resources is the mean-field formalism. This has originally been introduced as a modeling tool. We have shown that it offered a remarkable tool to interpret the results of DNS (based on the unparametrized partial differential equations).

The natural candidate to investigate stellar interiors is then to rely on an anelastic formulation of the problem. This allows to to take into account the variation of the reference density with depth. Such approaches have been used for example by Browning et al. (2006), Browning (2010) in the solar and stellar context.

The Boussinesq approach offers an intermediate description. It is free of parametrisation, but lacks the description of effects indiced by stratification. It is however striking that differential rotation profiles obtained with this approach (e.g. Browning, 2010) are extremely similar to those obtained using Boussinesq models. In fact, since our results were published, Gastine et al. (2012) were able to reproduce a similar set of transitions 

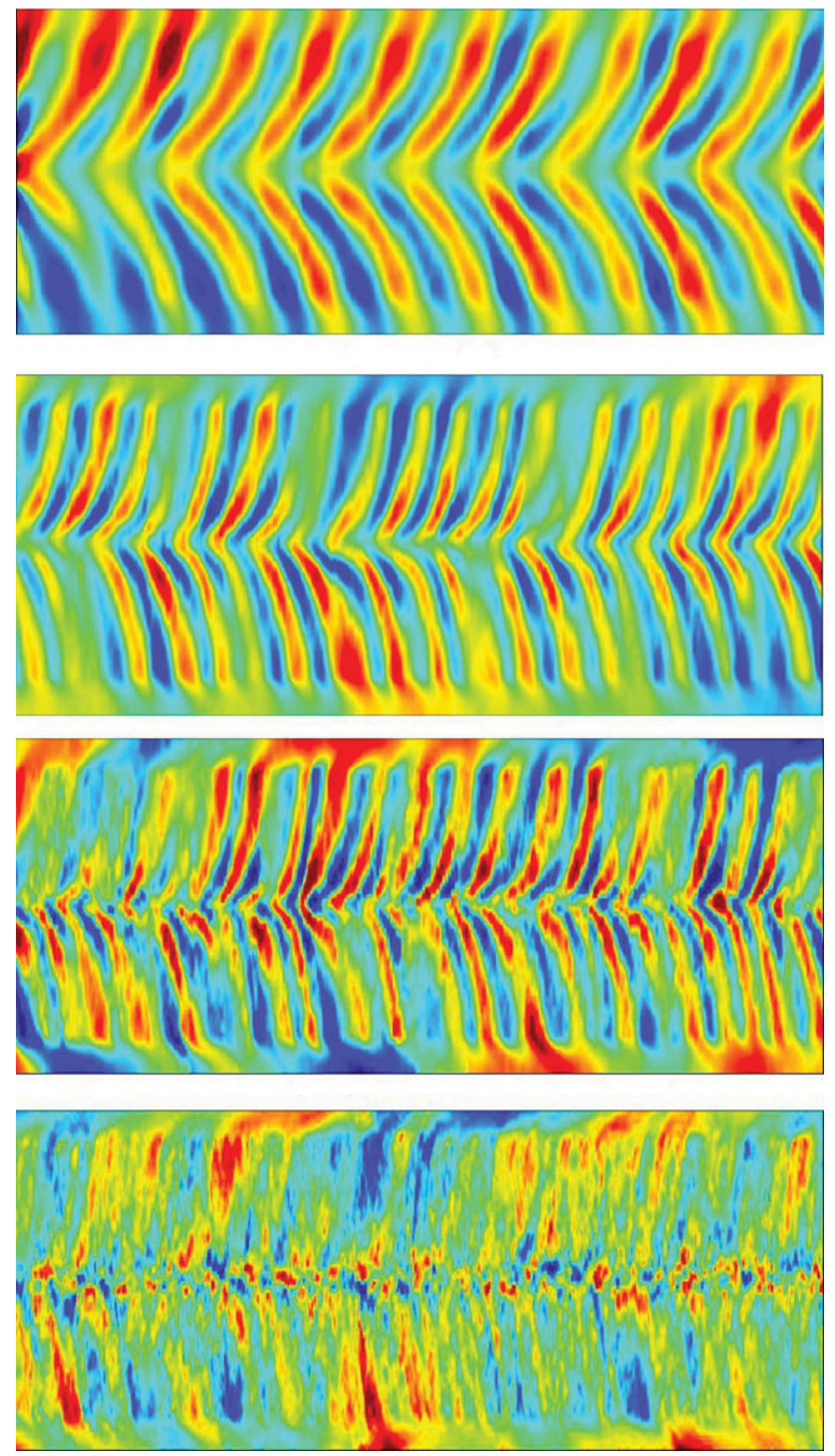

Figure 10. The coherence of the butterfly diagram in DNS appears directly related to the magnetic Reynolds number $\mathrm{Rm}=\mathrm{RoPm} / \mathrm{E}=U_{\mathrm{rms}} L / \eta$. Coherence is lost as the magnetic Reynolds number is increased. Here the diagrams respectively correspond (from top to bottom) to $\mathrm{Rm}=75,142,270,460$.

as those reported in section 5 but using an anelastic model. They observed that the local Rossby number was the controlling parameter in anelastic models as well.

\section{Conclusion}

We have shown that information on stellar dynamics can be gained from direct numerical models relying on a simple Boussinesq formulation. Boussinesq models offer a simple and flexible tool to investigate stellar dynamics and dynamo action in stars. As any model they require careful interpretation and cannot account for stratification effects. 
Boussinesq models provide a useful tool to understand how the magnetic field behaviour is affected by the controlling parameters.

Besides the bistability we reported, and which was since then also observed in anelastic models, could be relevant to low mass stars. Spectropolarimetric and spectroscopic observations of two groups of very low mass fully-convective stars sharing similar stellar parameters but generating radically different types of magnetic fields. This bi-stability could be the equivalent, albeit involving inertial rather than viscous effects, to the weakfield versus strong-field bistability predicted for the geodynamo (see Morin et al., 2011).

\section{Acknowledgments}

The ideas and results presented in this review were developed over the last few years in collaboration with co-workers, in particular: Vincent Morin, Laure Goudard, Julien Morin and Jean-François Donati.

\section{References}

Browning, M. K. M. S., Miesch, A. S., \& Brun, J. Toomre, Astrophys. J. L. 648, L157-L160 (2006)

Browning M., Proceedings IAU Symposium 271 (2010)

Dormy E., Soward A.M. (Eds), Mathematical Aspects of Natural dynamos, CRC-press (2007)

Gastine, T. L. \& Duarte, J. Wicht, A\&A 546, A19 (2012)

Gilman P. A., Astr. Phys. Sup. Ser. 53, 243-268 (1983)

Glatzmaier G. A. \& P. H. Roberts, Nature 377, 203-209 (1995)

Goudard L. \& E. Dormy, Europhysics Letters (EPL), 83, 59001 (2008)

Morin V. \& E. Dormy, International Journal of Modern Physics B, 23, 5467-5482 (2009)

Morin, J. E. Dormy, M. Schrinner, \& J.-F. Donati, Monthly Notices of the Royal Astronomical Society: Letters, 418, 1, L133-L137 (2011)

Roberts P. H., Phil. Trans. Roy. Soc., A 272, 1230, 663-698 (1972)

Schrinner, M., Rädler, K.-H., Schmitt, D., Rheinhardt, M., \& Christensen, U. R. Geophys. Astrophys. Fluid Dyn. 101 81-116 (2007)

Schrinner, M., Schmitt, D., Cameron, R., \& Hoyng, P., Geophys. J. Int., 182, 675 (2010)

Schrinner, M. L. \& Petitdemange E. Dormy, A\&SA 530, A140 (2011)

Schrinner M., A $G A$ 533, A108 (2011)

Schrinner M., L. \& Petitdemange, E. Dormy,ApJ Astrophysical Journal (ApJ) 752, 121 (2012)

Simitev, R., Busse, F. H. \& Kosovichev, A. G., Procs 2010 CTR Summer Program, P. Moin (ed.), pp. 475-484, Stanford University (2010)

Simitev, R., Busse, F. H., Phys. Scr., 86, 018409 (2012)

Tobias S. \& N. O. Weiss in Mathematical Aspects of Natural dynamos, Dormy E., Soward A. M. (Eds), CRC-press (2007)

Zhang K. \& Schubert G., Rep. Prog. Phys, 69, 1581-1605 (2006) 